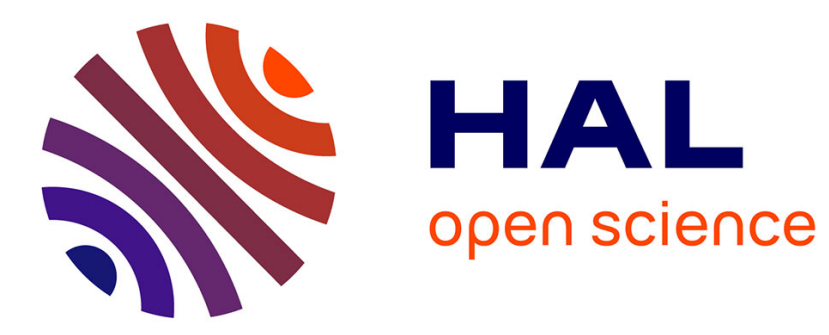

\title{
Moving horizon state estimation for linear discrete-time singular systems
}

Boulaïd Boulkroune, Mohamed Darouach, Michel Zasadzinski

\section{To cite this version:}

Boulaïd Boulkroune, Mohamed Darouach, Michel Zasadzinski. Moving horizon state estimation for linear discrete-time singular systems. IET Control Theory and Applications, 2010, 4 (3), pp.339-350. 10.1049/iet-cta.2008.0280 . hal-00461209

\section{HAL Id: hal-00461209 \\ https://hal.science/hal-00461209}

Submitted on $10 \mathrm{Jul} 2010$

HAL is a multi-disciplinary open access archive for the deposit and dissemination of scientific research documents, whether they are published or not. The documents may come from teaching and research institutions in France or abroad, or from public or private research centers.
L'archive ouverte pluridisciplinaire HAL, est destinée au dépôt et à la diffusion de documents scientifiques de niveau recherche, publiés ou non, émanant des établissements d'enseignement et de recherche français ou étrangers, des laboratoires publics ou privés. 


\title{
Moving horizon state estimation for linear discrete-time singular systems
}

\author{
B. Boulkroune, M. Darouach and M. Zasadzinski *†
}

\begin{abstract}
In this paper, the moving horizon recursive state estimator for linear singular systems is derived from the least squares estimation problem. It will be shown that this procedure yields the same state estimate as the Kalman filter for descriptor systems when the noises are Gaussian. The obtained results are applied to the state and the unknown inputs estimation for discrete-time systems with unknown inputs. A numerical example is presented to illustrate the proposed method.
\end{abstract}

Keywords : Moving horizon approach, singular systems, least squares estimation, unknown inputs estimation.

\section{Introduction}

Singular systems (or descriptor systems) arise in many areas of engineering including electrical networks, power systems, aerospace engineering and chemical processing. Since the late 1970s they have attracted the attention of many researchers and were introduced to describe the dynamics of certain linear systems for which the standard state space representation is not applicable. Various books and survey papers dealing with these systems have addressed the issues of solvability, controllability and observability, etc. The state estimation for singular systems has been treated by many authors [3], [7, [8], [5], [18, [17], 24], 25], [12] and [13]. Most of these works present the generalized Kalman filter as a solution for recursive state estimation problem. Among these works, we can cite the Kalman filtering obtained through a deterministic approach [7], 8], [12] and [13].

Investing the success of receding horizon control in the estimation of dynamic states and parameters, for linear and nonlinear systems, recent attention has been concentrated on the moving horizon estimation (MHE). The MHE method was proposed and successfully applied to various types of dynamical systems, in unconstrained linear systems [16], constrained linear systems [20, hybrid systems [2], hybrid systems with unknown inputs [19] and nonlinear systems [21].

The interest of the MHE is the possibility of dealing with a limited amount of data, instead of using all the information available from the beginning. In this framework the strategy of MHE is to transform the estimation problem into a quadratic program using a moving fixed-size estimation window. The fixed-size estimation window is necessary to bound the size of the quadratic program. Only a fixed amount of measurement data is used to solve the optimization problem, so that the oldest measurement sample is discarded as a new sample becomes available. Among the reasons of successes of MHE approach are the

\footnotetext{
${ }^{*}$ The authors are with Centre de Recherche en Automatique de Nancy (CRAN-UMR 7039) NancyUniversity - CNRS, IUT de Longwy, 186 rue de Lorraine, 54400 Cosnes et Romain, France.

${ }^{\dagger}$ Michel.Zasadzinski@iut-longwy.uhp-nancy.fr
} 
possibility of incorporating the equality and inequality constraints and also the reduced size of data used for estimation.

The objective of this paper is to present the moving horizon estimation (MHE) approach for singular linear systems. The obtained results will be used for the state and the unknown inputs estimation for the standard systems with unknown inputs. It extends the MHE developed for standard systems to singular systems. We will show that under some assumptions the obtained results are equivalent to the Kalman filtering for singular systems. In our knowledge this is the first work on the MHE for singular systems.

The paper is organized as follows. Section 2 formulates the problem. The full information estimation is presented in section 3. In section 4, the MHE for singular systems is derived. Application to simultaneous state and unknown inputs estimation for standard linear systems with unknown inputs is presented in section 5. Section 6 is devoted to a numerical example.

\section{Problem statement and assumptions}

Let us consider the following discrete-time linear singular system described as follows

$$
\begin{aligned}
E x_{k+1} & =F x_{k}+B u_{k}+w_{k} \\
z_{k} & =H x_{k}+v_{k}
\end{aligned}
$$

where $x_{k} \in \Re^{n}$ is the state vector, $u_{k} \in \Re^{q}$ is the known control input, $z_{k} \in \Re^{m}$ is the measured output, $w_{k} \in \Re^{n_{1}}$ is the state noise vector and $v_{k} \in \Re^{m}$ is the measurement noise vector. Matrices $E \in \Re^{n_{1} \times n}, F \in \Re^{n_{1} \times n}, B \in \Re^{n_{1} \times q}$ and $H \in \Re^{m \times n}$ are reals with appropriate dimensions. When $n_{1}=n, E$ is singular, i.e. $\operatorname{det}(E)=0$. We assume that the statistics of the random variables $x_{0}, w_{k}$ and $v_{k}$ to be unknown.

Based on the measure $z$ over time $(0, k)$ and initial state estimate $\bar{x}_{0}$, the estimate of $x_{k}$, denoted $\widehat{x}_{k \mid k}$ (the notation $*_{i \mid j}$ means that this is a discrete-time variable at time $i$ given information up to time $j$ ) is computed from the least squares problem formulation.

Our objective is to present the MHE approach for singular linear systems described by (1). The MHE attempts to preserve the old information by using a "information" window that slides over the measurements. In the full information estimation, we are using all the sequences of measurements $z_{0}, z_{1}, \ldots, z_{k}$ for finding the filtered estimates. The state is estimated from the horizon of the most recent $N+1$ output measurements that moves forward at each sampling time when a new measurement is available. The old information is incorporated using a startup estimate $\bar{x}_{k-N}$ that is calculated from old filtered states and a specific weight $P_{k-N \mid k-N}$.

Before closing this section, let us introduce the following assumption which will be used in this paper.

Assumption 1. We assume that matrix $\left[\begin{array}{c}E \\ H\end{array}\right]$ is of full column rank (see [8, [5, 18, 12]).

This assumption is generally used for the causal filtering.

\section{$3 \quad$ Full information estimation}

In this section, we present the full information state estimation approach. In this approach, all the sequences of measurements $z_{0}, z_{1}, \ldots, z_{k}$ are used for finding the filtered estimates. The estimation problem is treated as purely deterministic estimation one. 
The general description of the deterministic filtering problem for discrete-time singular system is performed using the following least squares formulation [1] (see p. 135)

$$
\min J_{0}\left(\left\{x_{0}\right\}\right)=\left\|e_{0}\right\|_{P_{0}^{-1}}^{2}+\left\|v_{0}\right\|_{V^{-1}}^{2}
$$

for $k=0$ and

$$
\min J_{k}\left(\left\{x_{i}\right\}_{i=0}^{k}\right)=\left\|e_{0}\right\|_{P_{0}^{-1}}^{2}+\sum_{i=0}^{k-1}\left\|w_{i}\right\|_{W^{-1}}^{2}+\sum_{j=0}^{k}\left\|v_{j}\right\|_{V^{-1}}^{2}
$$

where

$$
\begin{aligned}
e_{0} & =x_{0}-\bar{x}_{0}, \\
w_{k} & =E x_{k+1}-F x_{k}-B u_{k}, \\
v_{k} & =z_{k}-H x_{k} .
\end{aligned}
$$

for $k>0$, where $\|z\|_{A}^{2}=z^{T} A z$. The matrix $V^{-1}$ is a symmetric positive definite penalty matrix on the output filtered error, $W^{-1}$ is a symmetric positive definite penalty matrix on the estimated state noise, $P_{0}^{-1}$ is a symmetric positive definite penalty matrix on the initial state estimate error, and $\bar{x}_{0}$ is the initial state estimate at time $k=0$.

Before giving the solution to the problem stated in (2), we present the following lemma.

Lemma 1. [15] Consider matrices $\alpha, \beta, R$ and $x$ of appropriate dimensions with $R \geqslant 0$. The optimization problem

$$
\min _{x}(\alpha x-\beta)^{T} R(\alpha x-\beta)
$$

has a unique solution if and only if the matrix $\alpha^{T} R \alpha$ is nonsingular. In this case the optimal solution is given by $\widehat{x}=\left(\alpha^{T} R \alpha\right)^{-1} \alpha^{T} R \beta$.

The optimal solution for the problem (2) is given by the following theorem.

Theorem 1. Under Assumption 1, the estimate $\widehat{x}_{k \mid k}$ that minimizes the cost function $J_{k}$ and the weighting matrix $P_{k \mid k}$ are given by

$$
\begin{aligned}
& \widehat{x}_{k \mid k}=\left[\begin{array}{ll}
I_{n} & \underbrace{0}_{k} \ldots
\end{array}\right]\left(\Xi_{k}+Q_{k}\right)^{-1} g_{k} \mathfrak{B}_{k} \\
& P_{k \mid k}^{-1}=\left[\begin{array}{ll}
I_{n} & \underbrace{0 \ldots}_{k} \ldots
\end{array}\right]\left(\Xi_{k}+Q_{k}\right)^{-1}\left[\begin{array}{c}
I_{n} \\
k \\
\vdots \\
0
\end{array}\right]
\end{aligned}
$$

for $k>0$, where

$$
\begin{aligned}
& P_{0 \mid 0}=\left(P_{0}+H^{T} V^{-1} H\right)^{-1}, \quad \mathfrak{B}_{i}=\left[\begin{array}{llllll}
\mathcal{Z}_{i}^{T} & \mathcal{Z}_{i-1}^{T} & \cdots & \mathcal{Z}_{2}^{T} & \mathcal{Z}_{1}^{T} & \mathbb{Z}_{0}^{T}
\end{array}\right]^{T}, \\
& Q_{i}=\left[\begin{array}{cc}
\Sigma^{T} \Omega \Sigma & 0 \\
0 & Q_{i-1}
\end{array}\right], \quad \Xi_{i}=\left[\begin{array}{cc}
0 & \Sigma^{T} \Omega \alpha_{i} \\
\alpha_{i}^{T} \Omega \Sigma & \Xi_{i-1}+\alpha_{i}^{T} \Omega \alpha_{i}
\end{array}\right], \quad g_{i}=\left[\begin{array}{cc}
\Sigma^{T} \Omega & 0 \\
\alpha_{i}^{T} \Omega & g_{i-1}
\end{array}\right], \\
& Q_{0}=\Sigma_{0}^{T} \Omega_{0} \Sigma_{0}, \quad \Xi_{0}=0, \quad g_{0}=\Sigma_{0}^{T} \Omega_{0}, \quad \Omega=\left[\begin{array}{cc}
W^{-1} & 0 \\
0 & V^{-1}
\end{array}\right]>0, \quad \Sigma=\left[\begin{array}{l}
E \\
H
\end{array}\right], \\
& \mathbb{A}=\left[\begin{array}{c}
-F \\
0
\end{array}\right], \quad \mathcal{Z}_{i}=\left[\begin{array}{c}
B u_{i-1} \\
z_{i}
\end{array}\right] \quad \text { for } \quad 1 \leqslant i \leqslant k, \quad \mathbb{Z}_{0}=\left[\begin{array}{c}
\bar{x}_{0} \\
z_{0}
\end{array}\right],
\end{aligned}
$$

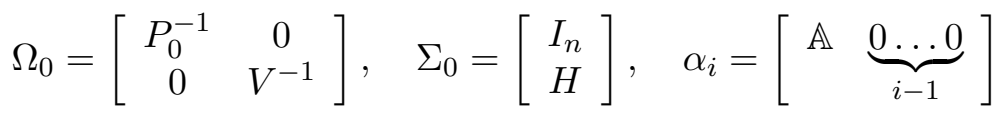

and $\alpha_{0}=0$. 
Proof. The discrete-time linear singular system (1) can be rewritten as follows

$$
\left[\begin{array}{c}
E \\
H
\end{array}\right] x_{k+1}-\left[\begin{array}{c}
F \\
0
\end{array}\right] x_{k}-\left[\begin{array}{c}
B u_{k} \\
z_{k+1}
\end{array}\right]=\left[\begin{array}{c}
w_{k} \\
-v_{k+1}
\end{array}\right]
$$

or equivalently as

$$
\Sigma x_{k+1}+\mathbb{A} x_{k}-\mathcal{Z}_{k+1}=\left[\begin{array}{c}
w_{k} \\
-v_{k+1}
\end{array}\right] \text {. }
$$

Then, for all $k \geqslant 0$, the minimization of $J_{k}$ can be written as follows

$$
\min _{\mathfrak{X}_{k}}\left(\mathfrak{A}_{k} \mathfrak{X}_{k}-\mathfrak{B}_{k}\right)^{T} \mathfrak{R}_{k}\left(\mathfrak{A}_{k} \mathfrak{X}_{k}-\mathfrak{B}_{k}\right)
$$

where

$$
\mathfrak{A}_{k}=\left[\begin{array}{ccccc}
\Sigma & \mathbb{A} & 0 & 0 & 0 \\
0 & \ddots & \ddots & 0 & 0 \\
0 & 0 & \Sigma & \mathbb{A} & 0 \\
0 & 0 & 0 & \Sigma & \mathbb{A} \\
0 & 0 & 0 & 0 & \Sigma_{0}
\end{array}\right], \quad \mathfrak{R}_{k}=\left[\begin{array}{cccc}
\Omega & 0 & 0 & 0 \\
0 & \ddots & 0 & 0 \\
0 & 0 & \Omega & 0 \\
0 & 0 & 0 & \Omega_{0}
\end{array}\right], \quad \mathfrak{X}_{k}=\left[\begin{array}{c}
x_{k} \\
x_{k-1} \\
\vdots \\
x_{0}
\end{array}\right]
$$

The solution to (7) using Lemma 1 is given by

$$
\widehat{\mathfrak{X}}_{k \mid k}=\left(\mathfrak{A}_{k}^{T} \mathfrak{R}_{k} \mathfrak{A}_{k}\right)^{-1} \mathfrak{A}_{k}^{T} \mathfrak{R}_{k} \mathfrak{B}_{k}
$$

This solution is unique if and only if $\mathfrak{A}_{k}^{T} \mathfrak{R}_{k} \mathfrak{A}_{k}$ is invertible. From invertibility of $\mathfrak{R}_{k}$, $\mathfrak{A}_{k}^{T} \mathfrak{R}_{k} \mathfrak{A}_{k}$ is invertible if and only if $\mathfrak{A}_{k}$ is a full column rank matrix. This condition is satisfied under Assumption 1.

For $k=0$, we have

$$
\begin{aligned}
\widehat{x}_{0 \mid 0} & =\left(\mathfrak{A}_{0}^{T} \mathfrak{R}_{0} \mathfrak{A}_{0}\right)^{-1} \mathfrak{A}_{0}^{T} \mathfrak{R}_{0} \mathfrak{B}_{0} \\
& =\left(\Sigma_{0}^{T} \Omega_{0} \Sigma_{0}\right)^{-1} \Sigma_{0}^{T} \Omega_{0} \mathbb{Z}_{0} \\
& =\left(\Xi_{0}+Q_{0}\right)^{-1} g_{0} \mathfrak{B}_{0}
\end{aligned}
$$

and

$$
\begin{aligned}
P_{0 \mid 0} & =\left(\Xi_{0}+Q_{0}\right)^{-1} \\
& =\left(P_{0}+H^{T} V^{-1} H\right)^{-1} .
\end{aligned}
$$

So, the initial step is verified. Now let

$$
\widehat{\mathfrak{X}}_{k \mid k}=\left[\begin{array}{c}
\widehat{x}_{k \mid k} \\
\widehat{\mathfrak{X}}_{k-1 \mid k}
\end{array}\right], \quad \Xi_{k}+Q_{k}=\mathfrak{A}_{k}^{T} \mathfrak{R}_{k} \mathfrak{A}_{k}, \quad g_{i}=\mathfrak{A}_{k}^{T} \mathfrak{R}_{k}
$$

then $\widehat{x}_{k \mid k}$ is given by

$$
\widehat{x}_{k \mid k}=\left[\begin{array}{lll}
I_{n} & \underbrace{0}_{k} \ldots & 0
\end{array}\right]\left(\Xi_{k}+Q_{k}\right)^{-1} g_{k} \mathfrak{B}_{k} .
$$

Define

$$
\mathfrak{B}_{k}=\left[\begin{array}{c}
\mathcal{Z}_{k} \\
\mathfrak{B}_{k-1}
\end{array}\right]
$$


we obtain

$$
\Xi_{k}+Q_{k}=\left[\begin{array}{cc}
\Sigma^{T} \Omega \Sigma & \Sigma^{T} \Omega \alpha_{k} \\
\alpha_{k}^{T} \Omega \Sigma & \Xi_{k-1}+Q_{k-1}+\alpha_{k}^{T} \Omega \alpha_{k}
\end{array}\right]
$$

Let

$$
\left(\Xi_{k}+Q_{k}\right)^{-1}=\left[\begin{array}{ll}
P_{11, k} & P_{12, k} \\
P_{21, k} & P_{22, k}
\end{array}\right]
$$

then we have

$$
\left[\begin{array}{c}
\widehat{x}_{k \mid k} \\
\widehat{\mathfrak{X}}_{k-1 \mid k}
\end{array}\right]=\left[\begin{array}{cc}
P_{11, k} & P_{12, k} \\
P_{21, k} & P_{22, k}
\end{array}\right]\left[\begin{array}{cc}
\Sigma^{T} \Omega & 0 \\
\alpha_{k}^{T} \Omega & g_{k-1}
\end{array}\right]\left[\begin{array}{c}
\mathcal{Z}_{k} \\
\mathfrak{B}_{k-1}
\end{array}\right]
$$

and thus

$$
\widehat{x}_{k \mid k}=\left[\begin{array}{ll}
P_{11, k} \Sigma^{T} \Omega+P_{12, k} \alpha_{k}^{T} \Omega & P_{12, k} g_{k-1}
\end{array}\right]\left[\begin{array}{c}
\mathcal{Z}_{k} \\
\mathfrak{B}_{k-1}
\end{array}\right] .
$$

Notice that we only need to calculate $P_{11, k}$ and $P_{12, k}$. After some algebraic manipulations, we obtain

$$
\begin{aligned}
& P_{11, k}^{-1}=\Sigma^{T}\left(\Omega^{-1}+\alpha_{k}\left(\Xi_{k-1}+Q_{k-1}\right)^{-1} \alpha_{k}^{T}\right)^{-1} \Sigma \\
& P_{12, k}=-P_{11, k} \Sigma^{T}\left(\Omega^{-1}+\alpha_{k}\left(\Xi_{k-1}+Q_{k-1}\right)^{-1} \alpha_{k}^{T}\right)^{-1} \alpha_{k}\left(\Xi_{k-1}+Q_{k-1}\right)^{-1}
\end{aligned}
$$

and the solution of (7) is given by

$$
\widehat{x}_{k \mid k}=\left(P_{11, k} \Sigma^{T} \Omega+P_{12, k} \alpha_{k}^{T} \Omega\right) \mathcal{Z}_{k}+P_{12, k} g_{k-1} \mathfrak{B}_{k-1}
$$

which is equivalent to

$$
\begin{aligned}
\widehat{x}_{k \mid k}=P_{11, k} \Sigma^{T} & \left(I_{n_{1}+m}-\left(\Omega^{-1}+\alpha_{k}\left(\Xi_{k-1}+Q_{k-1}\right)^{-1} \alpha_{k}^{T}\right)^{-1} \alpha_{k}\left(\Xi_{k-1}+Q_{k-1}\right)^{-1} \alpha_{k}^{T}\right) \Omega \mathcal{Z}_{k} \\
& -P_{11, k} \Sigma^{T}\left(\Omega^{-1} \alpha_{k}\left(\Xi_{k-1}+Q_{k-1}\right)^{-1} \alpha_{k}^{T}\right)^{-1} \alpha_{k}\left(\Xi_{k-1}+Q_{k-1}\right)^{-1} g_{k-1} \mathfrak{B}_{k-1} .
\end{aligned}
$$

By using the values of $\alpha_{k}, \Xi_{k-1}, Q_{k-1}, g_{k-1}$ and $\mathfrak{B}_{k-1}$, we obtain

$$
\begin{aligned}
\widehat{x}_{k \mid k}=-P_{11, k} \Sigma^{T}\left(\Omega^{-1}\right. & \left.+\mathbb{A} P_{11, k-1} \mathbb{A}^{T}\right)^{-1} \mathbb{A} \widehat{x}_{k-1 \mid k-1} \\
& +P_{11, k} \Sigma^{T}\left(I_{n_{1}+m}-\left(\Omega^{-1}+\mathbb{A} P_{11, k-1} \mathbb{A}^{T}\right)^{-1} \mathbb{A} P_{11, k-1} \mathbb{A}^{T}\right) \Omega \mathcal{Z}_{k}
\end{aligned}
$$

with

$$
P_{11, k}^{-1}=\Sigma^{T}\left(\Omega^{-1}+\mathbb{A} P_{11, k-1}^{-1} \mathbb{A}^{T}\right)^{-1} \Sigma
$$

and we obtain

$$
\widehat{x}_{k \mid k}=P_{11, k} \Sigma^{T}\left(\Omega^{-1}+\mathbb{A} P_{11, k-1} \mathbb{A}^{T}\right)^{-1}\left(\mathcal{Z}_{k}-\mathbb{A} \widehat{x}_{k-1 \mid k-1}\right) .
$$

If we take $P_{k \mid k}=P_{11, k}$, we find $4 \mathrm{~b}$. This completes the proof. 


\section{Remark 1.}

1. When matrix $E$ is of full column rank, the system (1) can be transformed easily to a standard linear system and in this case we can apply the existing results for standard linear systems.

2. The optimal solution given by Theorem 1 is equivalent to the optimal solution given by [8, 5] and [13].

3. For $E=I_{n}$, the deterministic estimate of Theorem 1 collapses to the usual state space Kalman filter estimate obtained from stochastic reasoning [1] and [14] when $w_{k}$ and $v_{k}$ are zero mean white noises with variance matrices $W$ and $V$.

The relation between the solution of the deterministic formulation given by Theorem 1 and the Kalman filter expression obtained by stochastical reasoning can be obtained by using the following lemma (see [12] and [13]).

Lemma 2. [18] Let $R \in \Re^{n \times n}$ be nonsingular and $A \in \Re^{n \times p}$ be a full column rank matrix. Then $A^{T} R^{-1} A$ is invertible and we have

$$
\begin{aligned}
\left(A^{T} R^{-1} A\right)^{-1} & =-\left[\begin{array}{ll}
0 & I_{p}
\end{array}\right]\left[\begin{array}{cc}
R & A \\
A^{T} & 0
\end{array}\right]^{-1}\left[\begin{array}{c}
0 \\
I_{p}
\end{array}\right], \\
\left(A^{T} R^{-1} A\right)^{-1} A^{T} R^{-1} & =\left[\begin{array}{ll}
0 & I_{p}
\end{array}\right]\left[\begin{array}{cc}
R & A \\
A^{T} & 0
\end{array}\right]^{-1}\left[\begin{array}{c}
I_{n} \\
0
\end{array}\right] .
\end{aligned}
$$

The following section presents the MHE for singular systems.

\section{Moving horizon estimation approach}

The basic idea of MHE can be the dualization of the model predictive control problem with respect to certain assumption. The moving horizon estimation method attempts to minimize the estimated weighted error of the state and measurement noise back to the past subject to some equality or inequality constraints, if any.

The full information formulation uses all $k$ output measurements to determine $k$ state noise vectors and obtain the state estimate at time $k$. We can see that the problem size grows with time as the estimator processes more data. In the Kalman filter recursion, the optimal state estimation at time $k$ is determined recursively from the optimal state estimate and output measurements at time $k-1$. In the receding horizon formulation, the optimal state estimate at time $k$ is determined recursively from the optimal state at time $k-N$ and the most recent $N+1$ output measurements using the following least squares formulation

$$
\min J_{k}\left(\left\{x_{i}\right\}_{i=k-N}^{k}\right)=\left\|e_{k-N}\right\|_{P_{k-N}^{-1}}^{2}+\sum_{i=k-N}^{k-1}\left\|w_{i}\right\|_{W^{-1}}^{2}+\sum_{j=k-N}^{k}\left\|v_{j}\right\|_{V^{-1}}^{2}
$$

where

$$
\begin{aligned}
e_{k-N} & =x_{k-N}-\bar{x}_{k-N}, \\
w_{k} & =E x_{k+1}-F x_{k}-B u_{k}, \\
v_{k} & =z_{k}-H x_{k} .
\end{aligned}
$$


The startup value $\bar{x}_{k-N}$ is determined from the filtered optimal estimate computed $N+1$ time intervals in the past

$$
\bar{x}_{k-N}=P_{k-N} \Sigma^{T}\left(\Omega^{-1}+\mathbb{A} P_{k-N-1} \mathbb{A}^{T}\right)^{-1}\left(\mathcal{Z}_{k-N}-\mathbb{A} \widehat{x}_{k-N-1 \mid k-N-1}\right) .
$$

Here, the initial penalty matrix $P_{k-N}$ is the matrix $P_{k-N \mid k-N}$ given by (15).

Note that in the case where $k \leqslant N$, the state estimates are determined using Theorem 1. Under Assumption 1 the unicity of the optimal solution of min $J_{k}$ is ensured. The solution of problem (17) is given by the following theorem.

Theorem 2. Under Assumption 1, the MHE of the state vector at time $k$ given the output measurement between $k-N$ and $k$, is computed as

$$
\widehat{x}_{k \mid k}=\left[\begin{array}{ll}
I_{n} & 0
\end{array}\right]\left(\Xi_{N}+\widetilde{Q}_{N}^{k-N}\right)^{-1} \widetilde{g}_{N}^{k-N} \widetilde{\mathfrak{B}}_{N}^{k-N}
$$

with

$$
\begin{aligned}
\widetilde{\mathfrak{B}}_{N}^{k-N} & =\left[\begin{array}{c}
\mathcal{Z}_{k} \\
\widetilde{\mathfrak{B}}_{N-1}^{k-N}
\end{array}\right], \widetilde{Q}_{i}^{k-N}=\left[\begin{array}{cc}
\Sigma^{T} \Omega \Sigma & 0 \\
0 & \widetilde{Q}_{i-1}^{k-N}
\end{array}\right], \widetilde{g}_{i}^{k-N}=\left[\begin{array}{cc}
\Sigma^{T} \Omega & 0 \\
\alpha_{i}^{T} \Omega & \widetilde{g}_{i-1}^{k-N}
\end{array}\right], \\
\widetilde{\mathfrak{B}}_{0}^{k-N} & =\bar{x}_{k-N}, \widetilde{Q}_{0}^{k-N}=P_{k-N \mid k-N}^{-1}, \widetilde{g}_{0}^{k-N}=P_{k-N \mid k-N}^{-1}
\end{aligned}
$$

Proof. We apply the same idea as in the proof of Theorem 1. For all $k \geqslant N$, the problem (17) can be written as follows

$$
\min _{\mathfrak{X}_{k}}\left(\mathfrak{A}_{k} \mathfrak{X}_{k}-\widetilde{\mathfrak{B}}_{N}^{k-N}\right)^{T} \mathfrak{R}_{k}\left(\mathfrak{A}_{k} \mathfrak{X}_{k}-\widetilde{\mathfrak{B}}_{N}^{k-N}\right)
$$

where $\mathfrak{A}_{k}, \mathfrak{R}_{k}$ and $\mathfrak{X}_{k}$ are given in $(8)$ except that the index 0 of the initial matrices $\Sigma_{0}$, $\Omega_{0}$ and $x_{0}$ is replaced by $k-N$ with $\Omega_{k-N}=P_{k-N \mid k-N}^{-1}$ and $\Sigma_{k-N}=I_{n}$.

Using Assumption 1 and Lemma 1, the solution of problem 21) is given by

$$
\widehat{\mathfrak{X}}_{k \mid k}=\left(\mathfrak{A}_{k}^{T} \mathfrak{R}_{k} \mathfrak{A}_{k}\right)^{-1} \mathfrak{A}_{k}^{T} \mathfrak{R}_{k} \widetilde{\mathfrak{B}}_{N}^{k-N}
$$

For $k=k-N$, we obtain

$$
\begin{aligned}
\widehat{x}_{k-N \mid k-N} & =\left(\mathfrak{A}_{k-N}^{T} \mathfrak{R}_{k-N} \mathfrak{A}_{k-N}\right)^{-1} \mathfrak{A}_{k-N}^{T} \mathfrak{R}_{k-N} \widetilde{\mathfrak{B}}_{0}^{k-N} \\
& =\left(\Sigma_{k-N}^{T} \Omega_{k-N} \Sigma_{k-N}\right)^{-1} \Sigma_{k-N}^{T} \Omega_{k-N} \bar{x}_{k-N} \\
& =\bar{x}_{k-N} .
\end{aligned}
$$

Note that $\bar{x}_{k-N}$ is given by $(18)$, then we can rewrite $\widehat{x}_{k-N \mid k-N}$ as following

$$
\widehat{x}_{k-N \mid k-N}=\left[\begin{array}{ll}
I_{n} & 0
\end{array}\right]\left[\begin{array}{cc}
\Sigma^{T} \Omega \Sigma & \Sigma^{T} \Omega \alpha_{1} \\
\alpha_{1}^{T} \Omega \Sigma & (2,2)
\end{array}\right]^{-1}\left[\begin{array}{cc}
\Sigma^{T} \Omega & 0 \\
\alpha_{1}^{T} \Omega & \widetilde{g}_{0}^{k-N-1}
\end{array}\right]\left[\begin{array}{c}
\mathcal{Z}_{k-N} \\
\widehat{x}_{k-N-1 \mid k-N-1}
\end{array}\right]
$$

where $(2,2)=\alpha_{1}^{T} \Omega \alpha_{1}+\Xi_{0}+\widetilde{Q}_{0}^{k-N-1}$. This is equivalent to

$$
\widehat{x}_{k-N \mid k-N}=\left[\begin{array}{ll}
I_{n} & 0
\end{array}\right]\left(\Xi_{1}+\widetilde{Q}_{1}^{k-N-1}\right)^{-1} \widetilde{g}_{1}^{k-N-1} \widetilde{\mathfrak{B}}_{1}^{k-N-1}
$$

which verifies the initial step.

Since for $k-N>0$, in the full information estimation case, we have proved that $\widehat{x}_{k \mid k}$ in (4a) for all $k>0$ can be written in a recursive form, by the same manner we can deduce that

$$
\widehat{x}_{k \mid k}=\left[\begin{array}{ll}
I_{n} & 0
\end{array}\right]\left(\Xi_{N}+\widetilde{Q}_{N}^{k-N}\right)^{-1} \widetilde{g}_{N}^{k-N} \widetilde{\mathfrak{B}}_{N}^{k-N}
$$

which corresponds to 19 . 
This estimate will be shown to be the Kalman filter one when the noises are Gaussian. The following lemma gives the expression of the matrix $P_{k \mid k}$.

Lemma 3. The matrix $P_{k \mid k}$ can be computed, for all $N \geqslant 0$ and $k>N$, as follows

$$
P_{k \mid k}^{-1}=\Sigma^{T}\left(\Omega^{-1}+\alpha_{N+1}\left(\Xi_{N}+\widetilde{Q}_{N}^{k-N}\right)^{-1} \alpha_{N+1}^{T}\right)^{-1} \Sigma .
$$

Proof. See the appendix.

Theorem 3. In the stochastic case, if the noises are Gaussian, the state estimate $\widehat{x}_{k \mid k}$ computed in (19) is the one obtained at time $k$ by the Kalman filter for singular systems.

Proof. The proof is by induction. First, for $N=0$, the solution of the least squares formulation in (17) yields the following estimate for all $k \geqslant 0$

$$
\widehat{x}_{k \mid k}=\left[\begin{array}{ll}
I_{n} & 0
\end{array}\right]\left(\Xi_{1}+\widetilde{Q}_{1}^{k-1}\right)^{-1} \widetilde{g}_{1}^{k-1} \widetilde{\mathfrak{B}}_{1}^{k-1}
$$

then

$$
\widehat{x}_{k \mid k}=\left[\begin{array}{ll}
I_{n} & 0
\end{array}\right]\left[\begin{array}{cc}
\Sigma^{T} \Omega \Sigma & \Sigma^{T} \Omega \alpha_{1} \\
\alpha_{1}^{T} \Omega \Sigma & \alpha_{1}^{T} \Omega \alpha_{1}+\Xi_{0}+\widetilde{Q}_{0}^{k-1}
\end{array}\right]^{-1}\left[\begin{array}{cc}
\Sigma^{T} \Omega & 0 \\
\alpha_{1}^{T} \Omega & \widetilde{g}_{0}^{k-1}
\end{array}\right]\left[\begin{array}{c}
\mathcal{Z}_{k} \\
\widehat{x}_{k-1 \mid k-1}
\end{array}\right]
$$

and using the partitioned matrix inverse, we obtain

$$
\begin{aligned}
\widehat{x}_{k \mid k}=-P_{k \mid k} \Sigma^{T}\left(\Omega^{-1}+\alpha_{1}\left(\Xi_{0}+\widetilde{Q}_{0}^{k-1}\right)^{-1}\right. & \left.\alpha_{1}^{T}\right)^{-1} \alpha_{1}\left(\Xi_{0}+\widetilde{Q}_{0}^{k-1}\right)^{-1} \widetilde{g}_{0}^{k-1} \widehat{x}_{k-1 \mid k-1} \\
& +P_{k \mid k} \Sigma^{T}\left(\Omega_{0}+\alpha_{1}\left(\Xi_{0}+\widetilde{Q}_{0}^{k-1}\right)^{-1} \alpha_{1}^{T}\right)^{-1} \mathcal{Z}_{k}
\end{aligned}
$$

which is equivalent to

$$
\widehat{x}_{k \mid k}=P_{k \mid k} \Sigma^{T}\left(\Omega^{-1}+\mathbb{A} P_{k-1 \mid k-1} \mathbb{A}^{T}\right)^{-1}\left(\mathcal{Z}_{k}-\mathbb{A} \widehat{x}_{k-1 \mid k-1}\right)
$$

which corresponds to the state estimate obtained by the Kalman filter, for $k \geqslant 0$, in the stochastic case.

Now, by using Lemma 2 and Lemma 3 with the initial condition given in Theorem 1 , the state estimate at time instant $k$ given by 19 for a horizon length $N=M+1$ is

$$
\widehat{x}_{k \mid k}=\left[\begin{array}{ll}
I_{n} & 0
\end{array}\right]\left(\Xi_{M+1}+\widetilde{Q}_{M+1}^{k-M-1}\right)^{-1} \widetilde{g}_{M+1}^{k-M-1} \widetilde{\mathfrak{B}}_{M+1}^{k-M-1}
$$

and

$$
P_{k \mid k}^{-1}=\Sigma^{T}\left(\Omega^{-1}+\alpha_{M+2}\left(\Xi_{M+1}+\widetilde{Q}_{M+1}^{k-M-1}\right)^{-1} \alpha_{M+2}^{T}\right)^{-1} \Sigma .
$$

Note that this is equivalent to eqution $\mathrm{A}-2$. Also by performing the partitioned matrix inverse we obtain

$$
\begin{aligned}
\widehat{x}_{k \mid k}=- & P_{k \mid k} \Sigma^{T}\left(\Omega^{-1}+\alpha_{M+2}\left(\Xi_{M+1}+\widetilde{Q}_{M+1}^{k-M-1}\right)^{-1} \alpha_{M+2}^{T}\right)^{-1} \alpha_{M+2}\left(\Xi_{M+1}+\widetilde{Q}_{M+1}^{k-M-1}\right)^{-1} \\
& \times \widetilde{g}_{M+1}^{k-M-1} \widetilde{\mathfrak{B}}_{M+1}^{k-M-1}+P_{k \mid k} \Sigma^{T}\left(\Omega^{-1}+\alpha_{M+2}\left(\Xi_{M+1}+\widetilde{Q}_{M+1}^{k-M-1}\right)^{-1} \alpha_{M+2}^{T}\right)^{-1} \mathcal{Z}_{k} .
\end{aligned}
$$


We assume that relation $(19)$ is the Kalman filter result for a horizon $N=M$ and for all $k \geqslant M$, hence we can write

$$
\widehat{x}_{k-1 \mid k-1}=\left[\begin{array}{ll}
I_{n} & 0
\end{array}\right]^{T}\left(\Xi_{M}+\widetilde{Q}_{M}^{(k-1)-M}\right)^{-1} \widetilde{g}_{M}^{(k-1)-M} \widetilde{\mathfrak{B}}_{M}^{(k-1)-M} .
$$

After some algebraic manipulations, we obtain

$\widehat{x}_{k \mid k}=-P_{k \mid k} \Sigma^{T}\left(\Omega^{-1}+\mathbb{A} P_{k-1 \mid k-1} \mathbb{A}^{T}\right)^{-1} \mathbb{A} \widehat{x}_{k-1 \mid k-1}+P_{k \mid k} \Sigma^{T}\left(\Omega^{-1}+\mathbb{A} P_{k-1 \mid k-1} \mathbb{A}^{T}\right)^{-1} \mathcal{Z}_{k}$

which is is the estimate obtained by the Kalman filter of singular systems in the stochastic framework, for all $k \geqslant 0$ using Lemma 2 and Lemma 3 which prove the theorem.

\section{Remark 2.}

1. If we take $E=I_{n}, N=0$, the solution is equivalent to the recursive Kalman filter for standard linear systems.

2. When we take $E=I_{n}, N \neq 0$, we obtain the results that given in [16], which is the moving horizon estimation for standard linear systems.

\section{Application to simultaneous state and unknown inputs estimation for linear systems}

The problem of state estimation for standard linear systems with unknown inputs has been focused more attention. In practice, this problem is very important because there are many situations where some of the system inputs are unknown and inaccessible. No available knowledge of a priori information about the nature of the unknown inputs is required in many works [22], [23], [10], 11], [9], [7, 6], 4] and [5]. Note that the state and the unknown inputs are estimated using the state estimation for singular systems in [5].

The aim of this section is to apply the results of the previous sections to linear systems with unknown inputs. For the full information estimation approach, we will show that our solution is equivalent to the optimal solution given in [8, 5] obtained by stochastical reasoning. To apply the moving horizon estimation approach to linear systems with unknown inputs, the result of Theorem 2 will be used.

Consider the following time-invariant linear discrete system described by

$$
\begin{aligned}
x_{k+1} & =A x_{k}+B u_{k}+B_{d} d_{k}+w_{k} \\
z_{k} & =C x_{k}+v_{k}
\end{aligned}
$$

where $x_{k} \in \Re^{n_{1}}$ is the state vector, $u_{k} \in \Re^{q}$ is the known control input, $z_{k} \in \Re^{m}$ is the measured output, $d_{k} \in \Re^{p}$ is the unmeasurable disturbance or unknown input vector, $w_{k} \in \Re^{n_{1}}$ is the state noise vector, and $v_{k} \in \Re^{m}$ is the measurement noise vector. Matrices $A, B, B_{d}$ and $C$ are constant with appropriate dimensions. Note that we do not assume anything about the type of disturbances $w_{k}$ and $v_{k}$.

The objective is to estimate the state vector $x_{k}$ and the unknown inputs $d_{k}$ using the moving horizon state estimation, assuming no knowledge about the vector $d_{k}$. The following assumption are generally made in unknown inputs observers.

Assumption 2. We suppose that the following conditions are verified 
i) $\operatorname{rank}(C)=m$,

ii) $\operatorname{rank}\left(B_{d}\right)=p$,

iii) $p \leqslant m$,

iv) $\operatorname{rank}\left(C B_{d}\right)=\operatorname{rank}\left(B_{d}\right)=p$.

Now system (27) can be written in the following singular system form

$$
\begin{aligned}
E X_{k+1} & =F X_{k}+B u_{k}+w_{k} \\
z_{k} & =H X_{k}+v_{k}
\end{aligned}
$$

where

$$
\begin{aligned}
X_{k+1} & =\left[\begin{array}{c}
x_{k+1} \\
d_{k}
\end{array}\right], \\
E & =\left[\begin{array}{ll}
I_{n_{1}}-B_{d}
\end{array}\right], \quad F=\left[\begin{array}{ll}
A & 0
\end{array}\right], \quad H=\left[\begin{array}{ll}
C & 0
\end{array}\right] .
\end{aligned}
$$

The condition of rank of $\left[\begin{array}{c}E \\ H\end{array}\right]$ is verified by the Assumption 2 . This rank condition is verified since $[5]$

$$
\begin{aligned}
\operatorname{rank}\left(\left[\begin{array}{c}
E \\
H
\end{array}\right]\right) & =\operatorname{rank}\left(\left[\begin{array}{cc}
I_{n_{1}} & -B_{d} \\
C & 0
\end{array}\right]\right) \\
& =\operatorname{rank}\left(\left[\begin{array}{cc}
I_{n_{1}} & 0 \\
-C & I_{m}
\end{array}\right]\left[\begin{array}{cc}
I & -B_{d} \\
C & 0
\end{array}\right]\right) \\
& =\operatorname{rank}\left(\left[\begin{array}{cc}
I_{n_{1}} & -B_{d} \\
0 & C B_{d}
\end{array}\right]\right) \\
& =n_{1}+\operatorname{rank}\left(C B_{d}\right) \\
& =n_{1}+p .
\end{aligned}
$$

\subsection{Full information estimation approach}

In this section, we will present the application of the full information estimation approach to the simultaneous state and unknown inputs by using the results of Theorem 1 . We obtain the following estimates

$$
\begin{aligned}
\widehat{x}_{k+1 \mid k+1} & =\left[\begin{array}{ll}
I_{n_{1}} & 0
\end{array}\right]\left(\Xi_{k+1}+Q_{k+1}\right)^{-1} g_{k+1} \mathfrak{B}_{k+1}, \\
\widehat{d}_{k \mid k+1} & =\left[\begin{array}{lll}
0_{n_{1} \times p} & I_{p} & 0
\end{array}\right]\left(\Xi_{k+1}+Q_{k+1}\right)^{-1} g_{k+1} \mathfrak{B}_{k+1} .
\end{aligned}
$$

Notice that this estimator is given in a deterministic recursive way. The relation between our algorithm and the Kalman filtering with unknown inputs can be established as follows.

The term $P_{k+1 \mid k+1}$ may be interpreted as the degree of the uncertainty for estimation error $X_{k+1}-\widehat{X}_{k+1 \mid k+1}$ at step $k+1$. Then, the deterministic interpretation as degree of uncertainty for $P_{k+1 \mid k+1}$ parallels the stochastic interpretation as the covariance of the error $X_{k+1}-\widehat{X}_{k+1 \mid k+1}$ [5]. The weighting matrix $P_{k+1 \mid k+1}$ is partitioned as follows

$$
P_{k+1 \mid k+1}=\left[\begin{array}{cc}
P_{k+1 \mid k+1}^{x} & P_{k+1 \mid k+1}^{x d} \\
P_{k+1 \mid k+1}^{d x} & P_{k \mid k+1}^{d}
\end{array}\right]
$$


with $P_{k+1 \mid k+1}^{x d}=\left(P_{k+1 \mid k+1}^{d x}\right)^{T}$. Using (30) and $\left.4 \mathrm{~b}\right)$, we get

$$
P_{k+1 \mid k+1}^{-1}=\left[\begin{array}{cc}
\bar{P}_{k \mid k}^{-1}+C^{T} V^{-1} C & -\bar{P}_{k \mid k}^{-1} B_{d} \\
-B_{d}^{T} \bar{P}_{k \mid k}^{-1} & B_{d}^{T} \bar{P}_{k \mid k}^{-1} B_{d}
\end{array}\right]
$$

with $\bar{P}_{k \mid k}=W+A P_{k \mid k}^{x} A^{T}$.

Using the inverse of the partitioned matrix in relation $(33)$, we have

$$
\begin{aligned}
P_{k+1 \mid k+1}^{x} & =\left(\bar{P}_{k \mid k}^{-1}+C^{T} V^{-1} C-\bar{P}_{k \mid k}^{-1} B_{d}\left(B_{d}^{T} \bar{P}_{k \mid k}^{-1} B_{d}\right)^{-1} B_{d}^{T} \bar{P}_{k \mid k}^{-1}\right)^{-1}, \\
P_{k \mid k+1}^{d} & =\left(B_{d}^{T} C^{T}\left(V+C \bar{P}_{k \mid k} C^{T}\right)^{-1} C B_{d}\right)^{-1}, \\
P_{k+1 \mid k+1}^{x d} & =P_{k+1 \mid k+1}^{x} \bar{P}_{k \mid k}^{-1} B_{d}\left(B_{d}^{T} \bar{P}_{k \mid k}^{-1} B_{d}\right)^{-1}, \\
P_{k+1 \mid k+1}^{d x} & =P_{k \mid k+1}^{d} B_{d}^{T} \bar{P}_{k \mid k}^{-1}\left(\bar{P}_{k \mid k}^{-1}+C^{T} V^{-1} C\right)^{-1} .
\end{aligned}
$$

In section 3, we have proved that relation (4a) can be written as (see (16)

$$
\widehat{X}_{k+1 \mid k+1}=P_{k+1 \mid k+1} \Sigma^{T}\left(\Omega^{-1}+\mathbb{A} P_{k \mid k} \mathbb{A}^{T}\right)^{-1}\left(\mathcal{Z}_{k+1}-\mathbb{A} \widehat{X}_{k \mid k}\right) .
$$

After some algebraic manipulations, we find

$$
\begin{aligned}
& \widehat{x}_{k+1 \mid k+1}=\left(P_{k+1 \mid k+1}^{x}-P_{k+1 \mid k+1}^{x d} B_{d}^{T}\right)\left(W+A P_{k \mid k}^{x} A^{T}\right)^{-1}\left(A \widehat{x}_{k \mid k}+B u_{k}\right)+P_{k+1 \mid k+1}^{x} C^{T} V^{-1} z_{k+1} \\
& \widehat{d}_{k \mid k+1}=\left(P_{k+1 \mid k+1}^{d x}-P_{k \mid k+1}^{d} B_{d}^{T}\right)\left(W+A P_{k \mid k}^{x} A^{T}\right)^{-1}\left(A \widehat{x}_{k \mid k}+B u_{k}\right) \\
&+P_{k+1 \mid k+1}^{d x} C^{T} V^{-1} z_{k+1}
\end{aligned}
$$

which is the generalized Kalman filter for systems with unknown inputs obtained by [8, 5 .

\subsection{Moving horizon estimation approach}

In this section, we will give an optimal solution of the problem of the simultaneous state and unknown inputs estimation using the moving horizon estimation approach. The optimal estimate at time $k+1$ is determined recursively from the optimal estimate at time $k-$ $N+1$ and the most recent $N+1$ output measurements using the following least squares formulation

$$
\begin{aligned}
\min J_{k+1}\left(\left\{X_{i}\right\}_{i=k-N+1}^{k+1}\right)=\| X_{k-N+1}- & \bar{X}_{k-N+1} \|_{P_{k-N+1}^{-1}}^{2} \\
& +\sum_{i=k-N+1}^{k}\left\|w_{i}\right\|_{W^{-1}}^{2}+\sum_{j=k-N+1}^{k+1}\left\|v_{j}\right\|_{V^{-1}}^{2}
\end{aligned}
$$

where $w_{i}$ and $v_{j}$ are given by (28).

The startup value $\bar{X}_{k-N+1}$ is determined from the filtered optimal estimate computed $N+1$ time intervals in the past

$$
\bar{X}_{k-N+1}=P_{k-N+1} \Sigma^{T}\left(\Omega^{-1}+\mathbb{A} P_{k-N} \mathbb{A}^{T}\right)^{-1}\left(\mathcal{Z}_{k-N+1}-\mathbb{A} \widehat{X}_{k-N \mid k-N}\right) .
$$

Here, the initial penalty matrix $P_{k-N+1}$ is the matrix $P_{k-N+1 \mid k-N+1}$ given by (4b).

Note that in the case where $k+1 \leqslant N$, the estimates are determined using Theorem 1 . Under Assumption 2, the unicity of the optimal solution of min $J_{k+1}$ is guaranteed. The solution of problem (35) is given by the following theorem. 
Theorem 4. Under Assumption 2, the estimate of the state vector, the unknown inputs and the weighting matrix at time $k+1$ given output measurements up to time $k+1$ are computed as follows

$$
\begin{aligned}
\widehat{x}_{k+1 \mid k+1} & =\left[\begin{array}{ll}
I_{n_{1}} & 0
\end{array}\right]\left(\Xi_{N}+\widetilde{Q}_{N}^{k+1-N}\right)^{-1} \widetilde{g}_{N}^{k+1-N} \widetilde{\mathfrak{B}}_{N}^{k+1-N} \\
\widehat{d}_{k \mid k+1} & =\left[\begin{array}{lll}
0_{n_{1} \times p} & I_{p} & 0
\end{array}\right]\left(\Xi_{N}+\widetilde{Q}_{N}^{k+1-N}\right)^{-1} \widetilde{g}_{N}^{k+1-N} \widetilde{\mathfrak{B}}_{N}^{k+1-N} \\
P_{k+1 \mid k+1} & =\left[\begin{array}{ll}
I_{n_{1}+p} & 0
\end{array}\right]\left(\Xi_{N}+\widetilde{Q}_{N}^{k+1-N}\right)^{-1}\left[\begin{array}{c}
I_{n_{1}+p} \\
0
\end{array}\right]
\end{aligned}
$$

with

$$
\begin{aligned}
\widetilde{\mathfrak{B}}_{N}^{k-N} & =\left[\begin{array}{c}
\mathcal{Z}_{k} \\
\widetilde{\mathfrak{B}}_{N-1}^{k-N}
\end{array}\right], \quad \widetilde{Q}_{i}^{k-N}=\left[\begin{array}{cc}
\Sigma^{T} \Omega \Sigma & 0 \\
0 & \widetilde{Q}_{i-1}^{k-N}
\end{array}\right], \quad \widetilde{g}_{i}^{k-N}=\left[\begin{array}{cc}
\Sigma^{T} \Omega & 0 \\
\alpha_{i}^{T} \Omega & \widetilde{g}_{i-1}^{k-N}
\end{array}\right] \\
\widetilde{\mathfrak{B}}_{0}^{k-N} & =\left[\begin{array}{c}
\widehat{x}_{k-N} \\
\widehat{d}_{k-N-1}
\end{array}\right], \quad \widetilde{Q}_{0}^{k-N}=P_{k-N \mid k-N}^{-1}, \quad \widetilde{g}_{0}^{k-N}=P_{k-N \mid k-N}^{-1} .
\end{aligned}
$$

\section{Numerical example}

In this section, a numerical example is provided to show the performances of the proposed approach. The result given by Theorem 4 will be applied to a linear system with unknown inputs. Consider the example of the electromechanical actuator described in [26] and constituting of a direct current motor with an elastic coupling and the load shaft as shown in figure 1. This plant can be described by the following state-space model

$$
\begin{aligned}
& \dot{x}_{m}=A_{c} x_{m}+R_{c} u+M_{c} d \\
& y=C_{c} x_{m}
\end{aligned}
$$

where the index "c" stands for continuous-time and

$$
A_{c}=\left[\begin{array}{ccc}
\frac{-F_{m}}{J_{m}} & \frac{-k_{t}}{N_{1} J_{m}} & 0 \\
\frac{1}{N_{1}} & 0 & -1 \\
0 & \frac{k_{t}}{J_{c}} & \frac{-F_{c}}{J_{c}}
\end{array}\right], R_{c}=\left[\begin{array}{c}
\frac{k_{a}}{J_{m}} \\
0 \\
0
\end{array}\right], M_{c}=\left[\begin{array}{c}
0 \\
0 \\
\frac{-1}{J_{c}}
\end{array}\right], C_{c}=\left[\begin{array}{lll}
1 & 0 & 0 \\
0 & 0 & 1
\end{array}\right], x_{m}=\left[\begin{array}{c}
\omega_{m} \\
\Delta_{\Theta} \\
\omega_{c}
\end{array}\right] .
$$

The state variables are the motor shaft velocity $\omega_{m}$, the elastic torque $\Delta_{\Theta}$ and the motor shaft velocity $\omega_{c}$. The control input $u$ is the stator current $i_{e}$. The unknown disturbance $d$ is due to Coulomb frictions and load disturbance. The vector $w$ represents finite energy disturbances which affect both the control input and the second measurement. In the state-space description, $J_{m}$ and $J_{c}$ represent the motor and the load shaft inertia, $F_{m}$ and $F_{c}$ the motor and the load viscous friction coefficients, $k_{a}$ the motor torque constant, $k_{t}$ the coupling rigidity coefficient and $N_{1}$ the gear ratio. The numerical values of these parameters are $N_{1}=20, k_{a}=0.156\left[\mathrm{~m}^{2} \mathrm{~kg} \mathrm{sec}^{-2} \mathrm{~A}^{-2}\right], k_{t}=37.7\left[\mathrm{~m}^{2} \mathrm{~kg} \mathrm{sec}^{-2}\right], F_{m}=$ $0.0032\left[\mathrm{~m}^{2} \mathrm{~kg} \mathrm{sec}-1\right], F_{c}=0\left[\mathrm{~m}^{2} \mathrm{kgsec}^{-1}\right], J_{m}=0.00024\left[\mathrm{~m}^{2} \mathrm{~kg}\right], J_{c}=0.0825\left[\mathrm{~m}^{2} \mathrm{~kg}\right]$.

The continuous-time model is discretized using the "bilinear" method (see function "bilin" with option "tustin" in Matlab ${ }^{\circledR}$ with a time period $T=0.02$ second. The discretetime model of this plant is given by the following equations

$$
x_{k+1}=A x_{k}+B u_{k}+B_{d} d_{k}+w_{k}
$$




$$
z_{k}=C x_{k}+v_{k}
$$

with

$$
\begin{array}{rlrl}
A & =\left[\begin{array}{ccc}
0.7081 & -128.2948 & 1.2829 \\
0.0008 & 0.8513 & -0.0185 \\
0.0037 & 8.4597 & 0.9154
\end{array}\right], & B=\left[\begin{array}{c}
1110.2784 \\
0.5309 \\
2.4259
\end{array}\right], & \\
B_{d} & =\left[\begin{array}{c}
15.5509 \\
-0.2244 \\
23.2170
\end{array}\right], & C=\left[\begin{array}{ccc}
0.0085 & -0.6415 & 0.0064 \\
0.0000 & 0.0423 & 0.0096
\end{array}\right] .
\end{array}
$$

In the discrete-time model (39), the noise vectors $w_{k}$ and $v_{k}$ have been added to represent the modeling errors due to the discretization.

The weighting matrices were chosen as follows

$$
\begin{aligned}
& P_{0}^{x}=10^{3} \times\left[\begin{array}{lll}
3 & 0 & 0 \\
0 & 3 & 0 \\
0 & 0 & 3
\end{array}\right], P_{0}^{d}=3 \times 10^{3}, P_{0}^{x d}=0 \\
& W=\left[\begin{array}{ccc}
10000 & 0 & 0 \\
0 & 1000 & 0 \\
0 & 0 & 40
\end{array}\right], V=\left[\begin{array}{cc}
2000 & 0 \\
0 & 200
\end{array}\right] .
\end{aligned}
$$

The initial conditions for the system and for the filter have been chosen as $X_{0}=$ $\left[\begin{array}{ll}x_{0}^{T} & d_{0}^{T}\end{array}\right]^{T}=\left[\begin{array}{llll}1 & -1 & 0.1 & 0\end{array}\right]^{T}, \bar{X}_{0}=\left[\begin{array}{ll}\bar{x}_{0}^{T} & \bar{d}_{0}^{T}\end{array}\right]^{T}=\left[\begin{array}{llll}200 & 100 & 100 & 100\end{array}\right]^{T}$ and let us take the horizon length $N=5$.

Note that Assumption 2 is satisfied.

The simulation results based on Theorem 3 are given in figures $2-8$ where the actual values are in blue line and the estimated values are in red line. The actual and estimated values of the state $x$ are given in figures $2-4$ for the total duration of simulation, while the figures $5-7$ relate to only the period going up to one second. The actual and the estimated unknown inputs are given by figure 8 with the control input $u$ (in green).

In these simulations, the noises are of important magnitudes. The results show that the states are well estimated, however the input estimate is fluctuating around the true input, this is due to the importance of the noises and to the fact we have no any information about this input.

\section{Conclusion}

In this paper the moving horizon estimation method was extended to a discrete-time linear singular systems. The equivalence between this approach and the Kalman filter is shown in the Gaussian case. An application to simultaneous state and unknown inputs estimation for linear systems with unknown inputs is presented. A numerical example is given to illustrate the obtained results. The general case where the state and inputs are subjected to inequality constraints is currently under study.

\section{Acknowledgments}

This work is supported by the Ministry for the Culture, the Higher Education and Research of Luxembourg (BFR 04/117) and FNR (Fonds National de la Recherche) of Luxembourg. 


\section{Appendix : proof of Lemma (3)}

The proof can be obtained by induction. For $N=0$, we have

$$
P_{k \mid k}^{-1}=\Sigma^{T}\left(\Omega^{-1}+\alpha_{1}\left(\Xi_{0}+\widetilde{Q}_{0}^{k-1}\right)^{-1} \alpha_{1}^{T}\right)^{-1} \Sigma .
$$

Substituting the expressions of variables $\alpha_{1}, \Xi_{0}$ and $\widetilde{Q}_{0}$ into $\mathrm{A}-1$ gives

$$
P_{k \mid k}^{-1}=\Sigma^{T}\left(\Omega^{-1}+\mathbb{A} P_{k-1 \mid k-1} \mathbb{A}^{T}\right)^{-1} \Sigma
$$

The above difference equation is similar to the filtering Riccati equation for all $k>0$ given by [8, 5, 12] using Lemma 2. Assume that

$$
P_{j+N-1 \mid j+N-1}^{-1}=\Sigma^{T}\left(\Omega^{-1}+\alpha_{N}\left(\Xi_{N-1}+\widetilde{Q}_{N-1}^{j}\right)^{-1} \alpha_{N}^{T}\right)^{-1} \Sigma
$$

is verified for $k=j+N-1$.

For $k=j+N$ with all $j \geqslant 1$, we have

$$
P_{j+N \mid j+N}^{-1}=\Sigma^{T}\left(\Omega^{-1}+\alpha_{N+1}\left(\Xi_{N}+\widetilde{Q}_{N}^{j-1}\right)^{-1} \alpha_{N+1}^{T}\right)^{-1} \Sigma .
$$

Substituting the values of $\alpha_{N+1}, \Xi_{N}$ and $\widetilde{Q}_{N}^{j-1}$ into $\alpha_{N+1}\left(\Xi_{N}+\widetilde{Q}_{N}^{j-1}\right)^{-1} \alpha_{N+1}^{T}$ and by using the partitioned matrix inversion formula, we obtain

$$
\begin{aligned}
\alpha_{N+1}\left(\Xi_{N}+\widetilde{Q}_{N}^{j-1}\right)^{-1} \alpha_{N+1}^{T}= & \alpha_{1}\left(\Sigma^{T} \Omega \Sigma-\Sigma^{T} \Omega \alpha_{N}\right. \\
& \left.\times\left(\alpha_{N}^{T} \Omega \alpha_{N}+\Xi_{N-1}+\widetilde{Q}_{N-1}^{j}\right)^{-1} \alpha_{N}^{T} \Omega \Sigma\right)^{-1} \alpha_{1}^{T} .
\end{aligned}
$$

Using $(\mathrm{A}-3)$ in the above equation gives

$$
\alpha_{N+1}\left(\Xi_{N}+\widetilde{Q}_{N}^{j-1}\right)^{-1} \alpha_{N+1}^{T}=\alpha_{1} P_{j+N-1 \mid j+N-1} \alpha_{1}^{T} .
$$

Then, inserting (A-6) into (A-4) yields

$$
P_{j+N \mid j+N}^{-1}=\Sigma^{T}\left(\Omega^{-1}+\alpha_{1} P_{j+N-1 \mid j+N-1} \alpha_{1}^{T}\right)^{-1} \Sigma .
$$

This equation can be shown to be the filtering Riccati equation and this completes the proof.

\section{References}

[1] B. Anderson and J. Moore, Optimal Filtering. Englewood Cliffs, New Jersey: Prentice Hall, 1979.

[2] A. Bemporad, D. Mignone, and M. Morari, "Moving horizon estimation for hybrid systems and fault detection." in Proc. IEEE American Contr. Conf., San Diego, Canada, 1999, pp. 2471-2475.

[3] L. Dai, Singular Control Systems. Lecture Notes in Control and Information Sciences, Springer-Verlag, New York, 1989, vol. 118. 
[4] M. Darouach and M. Zasadzinski, "Unbiased minimum variance estimation for systems with unknown exogenous inputs," Automatica, vol. 33, pp. 717-719, 1997.

[5] M. Darouach, M. Zasadzinski, A. Bassong Onana, and S. Nowakowski, "Kalman filtering with unknown inputs via optimal state estimation of singular systems," Int. J. Syst. Sci., vol. 26, pp. 2015-2028, 1995.

[6] M. Darouach, M. Zasadzinski, and M. Boutayeb, "Extension of minimum variance estimation for systems with unknown inputs," Automatica, vol. 39, pp. 867-876, 2003.

[7] M. Darouach, M. Zasadzinski, and J. Y. Keller, "State estimation for discrete systems with unknown inputs using state estimation of singular systems," in Proc. IEEE American Contr. Conf., 1992, pp. 3014-3015.

[8] M. Darouach, M. Zasadzinski, and D. Mehdi, "State Estimation of Stochastic Singular Linear Systems," Int. J. Syst. Sci., vol. 24, no. 2, pp. 345-354, 1993.

[9] M. Darouach, M. Zasadzinski, and S. Xu, "Full-order observers for linear systems with unknown inputs," IEEE Trans. Aut. Contr., vol. 39, pp. 606-609, 1994.

[10] Y. Guan and M. Saif, "A novel approach to the design of unknown input observer." IEEE Trans. Aut. Contr., vol. 36, no. 5, pp. 632-635, 1991.

[11] M. Hou and P. Müller, "Design of observers for linear systems with unknown inputs," IEEE Trans. Aut. Contr., vol. 37, pp. 871-875, 1992.

[12] J. Y. Ishihara, J. C. T. Campos, and M. H. Terra, "Optimal Recursive Estimation for Discrete-time Descriptor Systems," in Proc. IEEE American Contr. Conf., Boston, U.S.A., 2004, pp. 189-193.

[13] J. Y. Ishihara, M. H. Terra, and J. C. T. Campos, "Optimal Recursive Estimation for Discrete-time Descriptor Systems," Int. J. Syst. Sci., vol. 36, no. 10, pp. 1-22, 2005.

[14] T. Kailath, A. Sayed, and B. Hassibi, Linear Estimation. Englewood Cliffs, New Jersey: Prentice Hall, 2000.

[15] C. Lawson and R. Hanson, Solving Linear Least Squares Problems. Englewood Cliffs, New Jersey: Prentice Hall, 1974.

[16] K. R. Muske and J. B. Rawlings, "Receding Horizon Recursive State Estimation," in Proc. IEEE American Contr. Conf., San Francisco, U.S.A., 1993, pp. 900-904.

[17] R. Nikoukhah, S. L. Campbell, and F. Delebecque, "Kalman Filtering for General Discrete-time Linear Systems," IEEE Trans. Aut. Contr., vol. 44, no. 10, pp. 1829 1839, 1999.

[18] R. Nikoukhah, A. S. Willsky, and B. C. Levy, "Kalman Filtering and Riccati Equations for Descriptor Systems," IEEE Trans. Aut. Contr., vol. 37, no. 9, pp. 1325-1342, 1992.

[19] L. Pina and M. A. Botto, "Simultaneous state and input estimation of hybrid systems with unknown inputs." Automatica, vol. 42, no. 5, pp. 755-762, 2006.

[20] C. V. Rao, J. B. Rawlings, and H. L. Jay, "Constrained Linear State Estimation A Moving Horizon Approach," Automatica, vol. 37, pp. 1619-1628, 2001. 
[21] C. V. Rao, J. B. Rawlings, and D. Q. Mayne, "Constrained state estimation for nonlinear discrete-time systems: Stability and moving horizon approximations," in IEEE Trans. Aut. Contr., vol. 48(2), 2003, pp. 246-258.

[22] S. H. Wang, E. J. Davidson, and P. Dorato, "Observing the states of systems with unmeasurable disturbances," IEEE Trans. Aut. Contr., vol. 20, no. 5, pp. 716-717, 1975 .

[23] F. Yang and R. W. Wilde, "Observers for linear systems with unknown inputs," IEEE Trans. Aut. Contr., vol. 33, no. 7, pp. 677-681, 1988.

[24] H. S. Zhang, L. Xie, and Y. C. Soh, "Optimal Recursive State Estimation for Singular Stochastic Discrete-Time Systems," in Proc. IEEE Conf. Decision 83 Contr., Tampa, Florida, U.S.A., 1998, pp. 2908-2913.

[25] — _ , "Optimal Recursive Filtering, Prediction and Smoothing for Singular Stochastic Discrete-Time Systems," IEEE Trans. Aut. Contr., vol. 44, no. 11, pp. 2154-2158, 1999.

[26] O. Malassé, M. Zasadzinski, C. Iung, M. Hayar and M. Darouach, "Comparison of robust loop shaping designs : an application to an electromechanical actuator," Proc. IEEE Conf. Contr. \& Applications, Glasgow, UK, 1994.

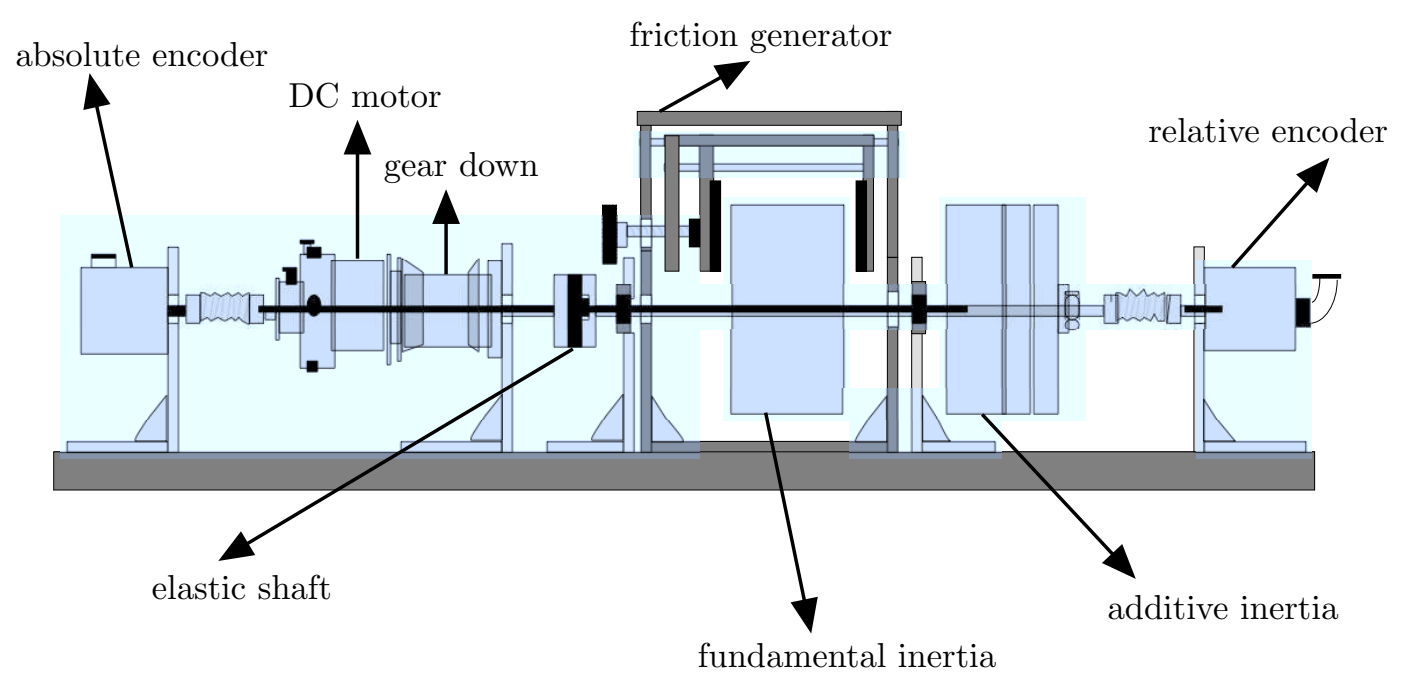

Figure 1: Electromechanical actuator. 


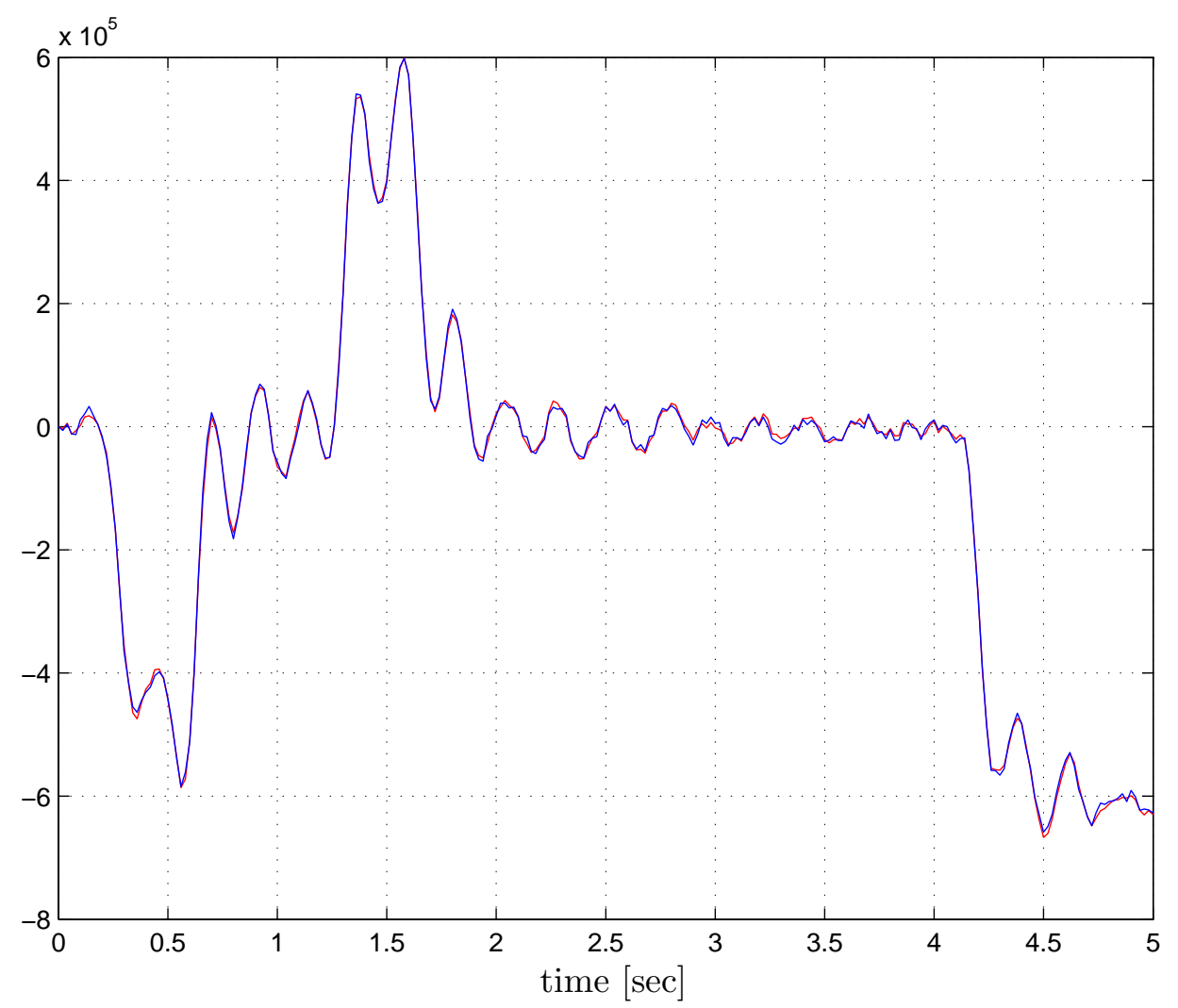

Figure 2: Actual (blue) and estimated (red) values of $x_{1}$.

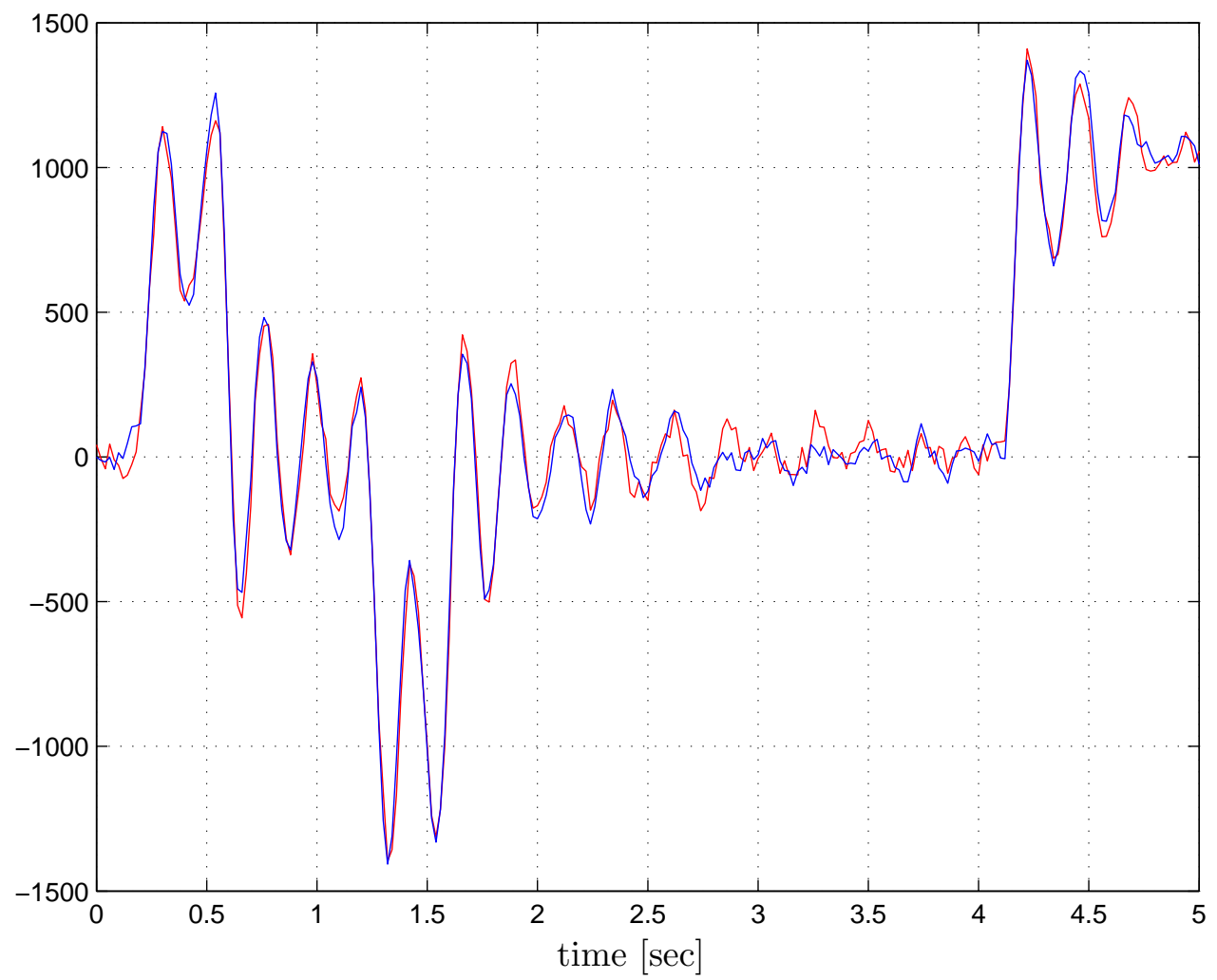

Figure 3: Actual (blue) and estimated (red) values of $x_{2}$. 


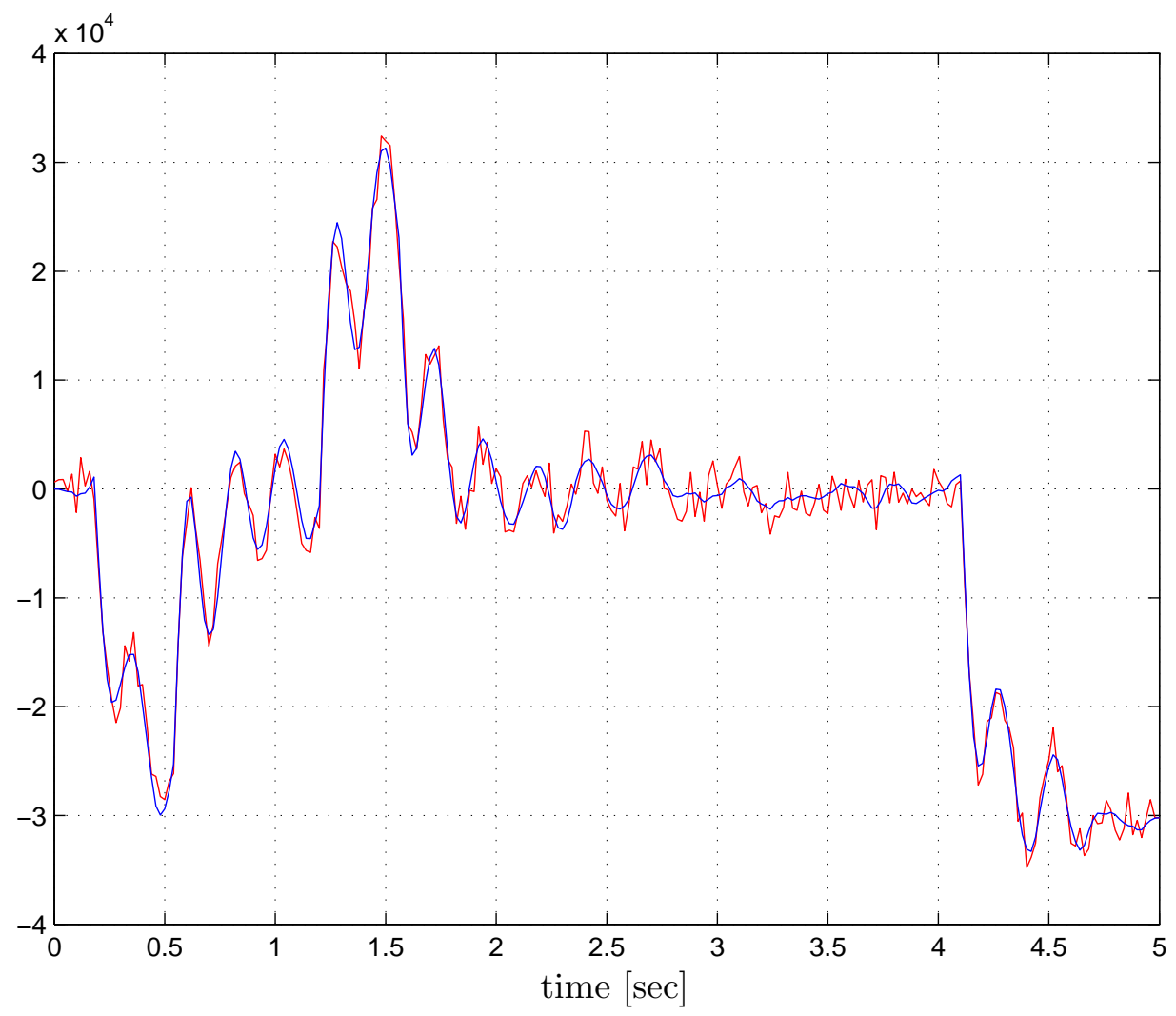

Figure 4: Actual (blue) and estimated (red) values of $x_{3}$.

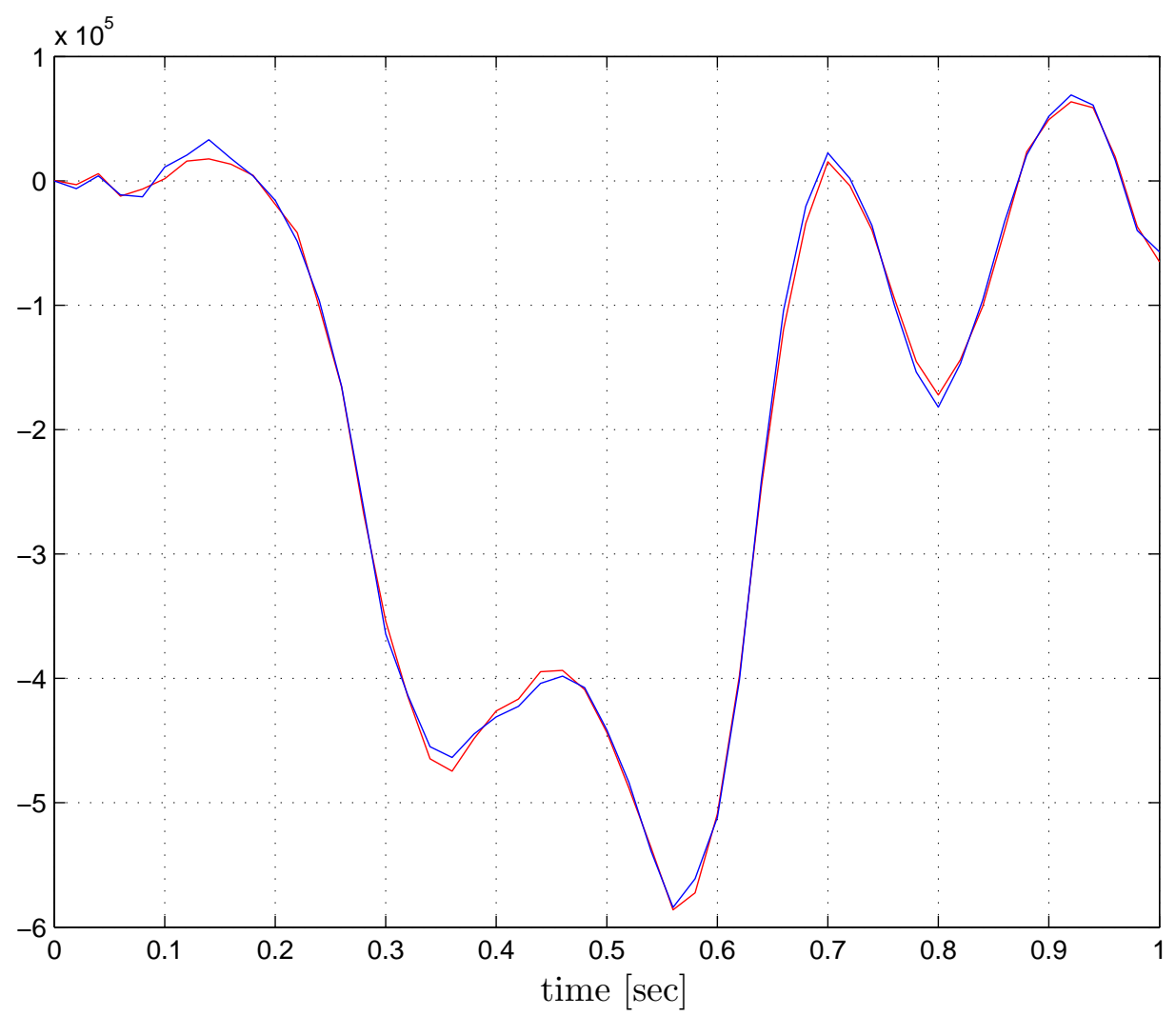

Figure 5: Actual (blue) and estimated (red) values of $x_{1}$. 


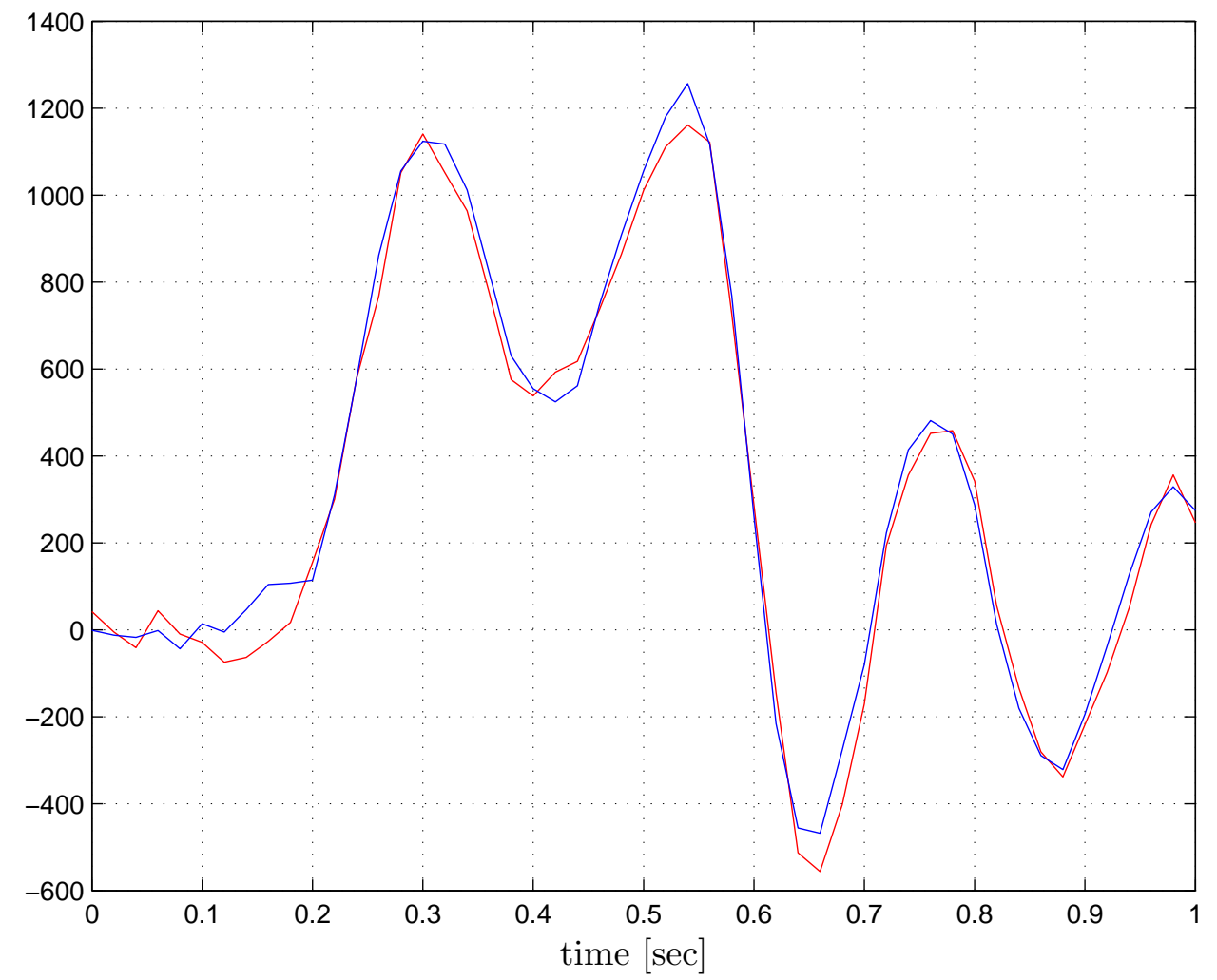

Figure 6: Actual (blue) and estimated (red) values of $x_{2}$.

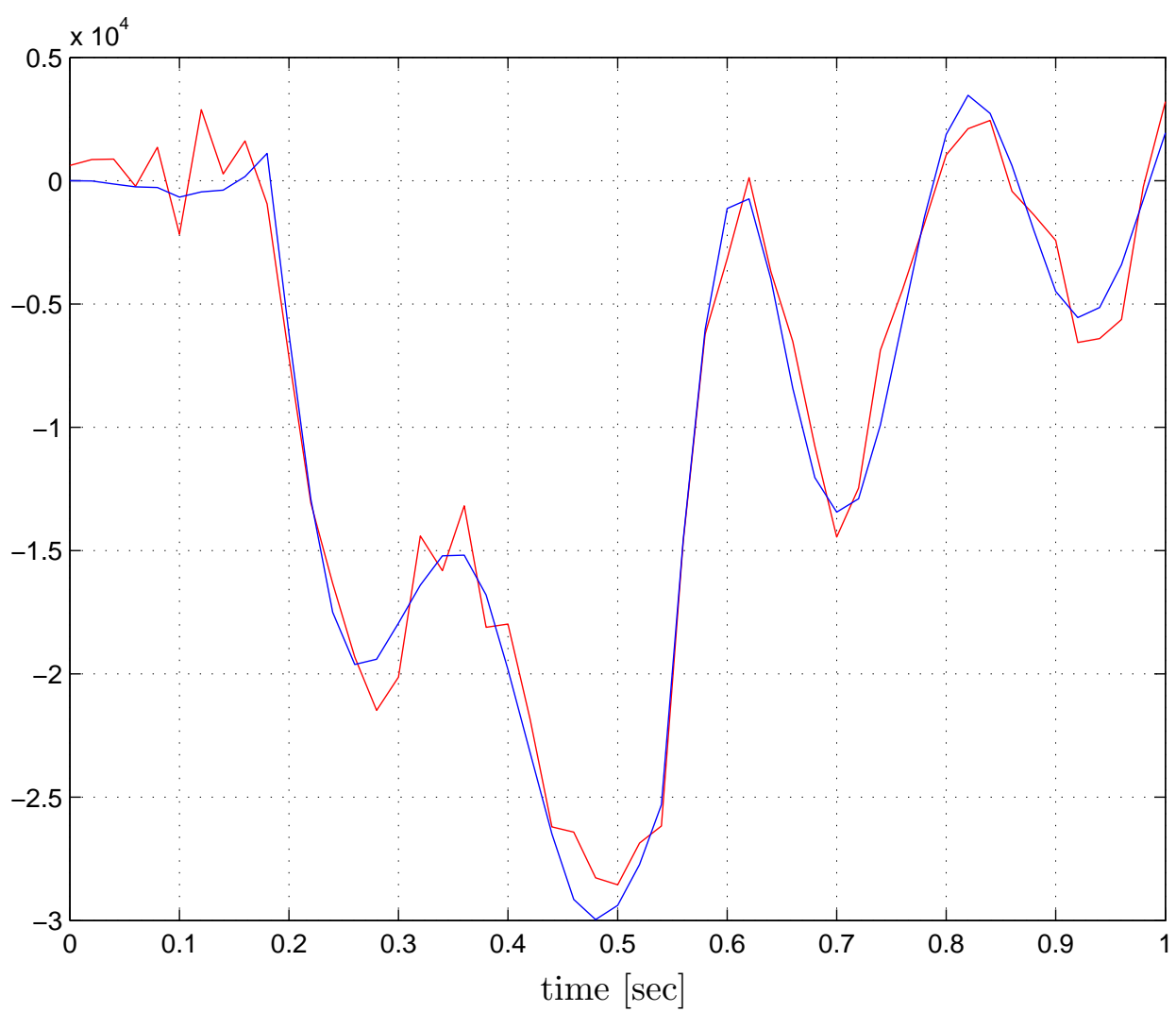

Figure 7: Actual (blue) and estimated (red) values of $x_{3}$. 


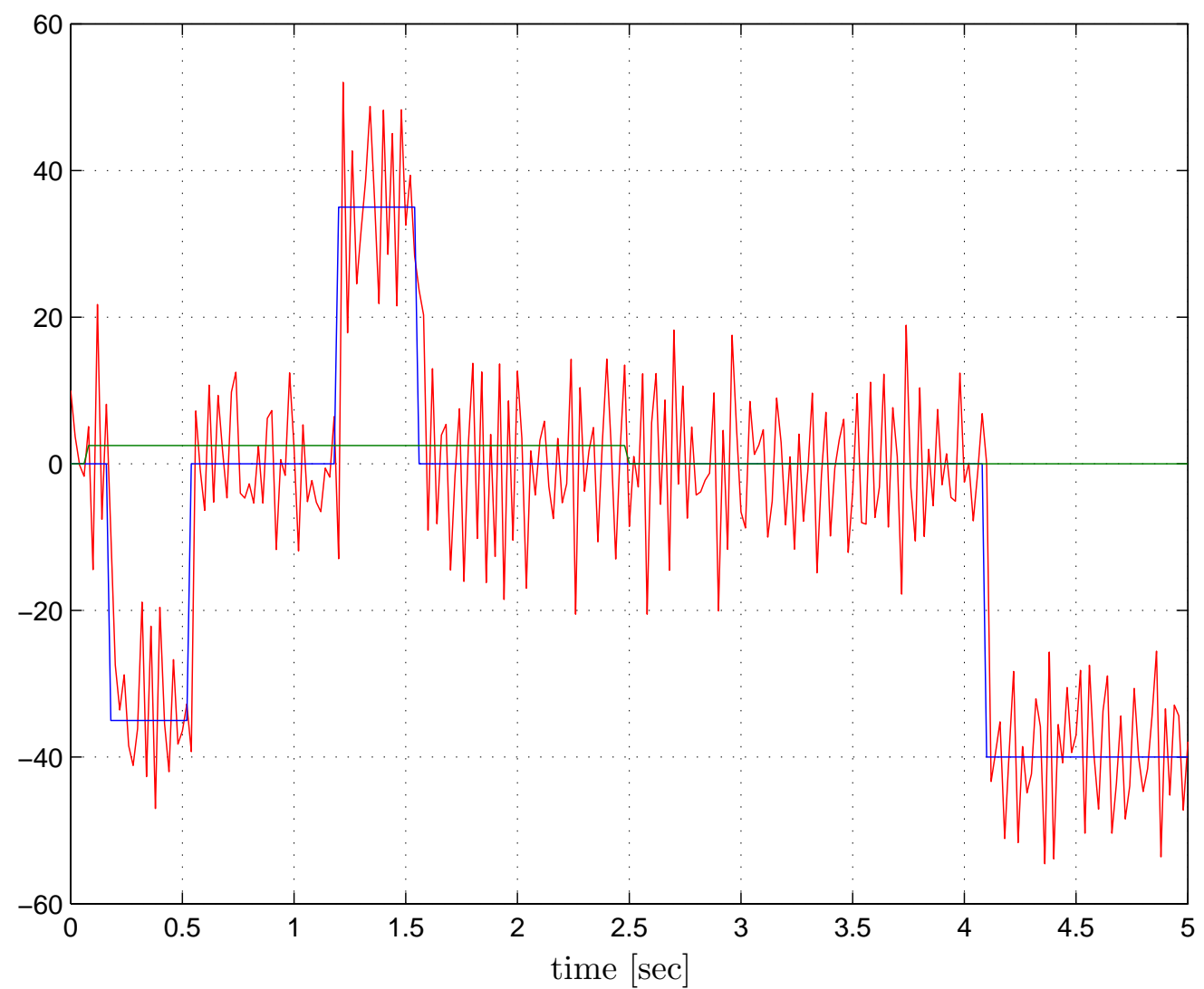

Figure 8: Actual (blue) and estimated unknown inputs $d_{k}$ (red), with control inut $u$ (green). 